\title{
RESIDENTS’ PERSPECTIVE
}

\section{CUA-AUA International Fellows Program: San Francisco 2018}

\author{
Taehyoung Lee, BSc, MD, FRCSC
}

McMaster University, Hamilton, ON, Canada

Cite as: Can Urol Assoc J 2018;12(8):229. htrp://dx.doi.org/10.5489/cuai.5503

\section{$\mathrm{T}$}

he CUA fosters excellence in urological practice through advocacy, education, research, and practicesupporting tools. Moreover, the CUA provides continuous professional development for Canadian urologists along the career-path continuum. The CUA-AUA International Fellows Program, for example, supports successful candidates to attend the annual AUA meeting. Residents and fellows from across the country are encouraged to participate in critical appraisal and professional dissemination of novel research presented during the meeting, and to discuss how the findings impact Canadian urological practice.

The AUA annual meeting is the largest gathering of urologists in the world, and it provides access to ground-breaking research, new guidelines, and the latest advances in urological medicine. Undoubtedly, navigating the countless plenary talks, poster presentations, and hands-on skill sessions can be a daunting process. Prior to the meeting this past May in San Francisco, there was a briefing where program faculty members provided important tips and guidance on how to remain organized and efficient at the conference.

At this year's meeting, there were several exciting presentations in the field of prostate cancer, including the amendment of the castrate-resistant prostate cancer (CRPC) guideline. ${ }^{1}$ The guideline now supports the use of apalutamide or enzalutamide in patients with non-metastatic CRPC at high risk of developing metastatic disease based on evidence from SPARTAN ${ }^{2}$ and PROSPER ${ }^{3}$ trials. Another impactful presentation came from the highly anticipated HORRAD trial, a prospective, multicentre, randomized controlled trial that randomized 432 patients with bony metastatic prostate cancer to androgen-deprivation therapy (ADT) plus external beam radiation therapy (EBRT) vs. ADT alone. ${ }^{4}$

The concept of primary local therapy for metastatic prostate cancer has recently been controversial. The potential benefits of such therapy include tumour debulking, which leads to increased efficacy of subsequent systemic treatment, delayed emergence of castrate resistance, prevention of primary tumour seeding or metastasis, and local symptomatic control. Previous evidence relied heavily on data from largely retrospective studies. The HORRAD trial aims to determine whether the addition of primary local therapy via EBRT has favourable impact on oncological outcomes. Interestingly, the overall survival with a median followup of 47 months (interquartile range [IQR] 36-68) did not show statistical difference between treatment and control groups (45 months vs. 43 months; hazard ratio [HR] 0.90; $p=0.356$ ). Although ADT plus EBRT did not achieve overall survival benefit, the subgroup analysis of patients with prostate-specific antigen (PSA) $<145 \mathrm{ng} / \mathrm{mL}$ (median PSA), $<5$ osseous metastatic lesions, and a Gleason score $<9$ showed trends in favour of ADT plus EBRT compared to ADT alone. However, again, statistical significance was not reached (HR 0.43; 95\% confidence interval [Cl] 0.17-1.05; $\mathrm{p}=0.063$ ).

While the HORRAD trial provides important insights on the role of primary local therapy in patients with high-volume, bony metastatic prostate cancer, the study population may not reflect the contemporary patient cohort with oligometastatic disease. Based on current evidence, there is no clinical role for primary local therapy for patients with high-volume metastatic prostate cancer outside of a trial setting. There are multiple ongoing prospective trials, such as STAMPEDE (Arm H), M1-MDACC, PEACE-1, g-RAMPP, and SWOG S1802, that may provide further guidance in similar disease settings.

At the meeting's conclusion, program participants had an opportunity to critically appraise numerous abstracts and discuss practical implications with peers, as well as faculty members. This provided an excellent avenue to consolidate my newly acquired knowledge and consider how this would impact my future practice. My experience at the 2018 AUA annual meeting was unquestionably enhanced by the CUA-AUA International Fellows Program, and I would like to thank my peers, sponsors, and the CUA for this incredible opportunity.

\section{References}

1. American Urological Association (2018). Castration-resistant prostate cancer: AUA guideline. Available at http://www.auanet.org/guidelines/castration-resistant-prostate-cancer-(2013-amended-2018). Accessed June 23, 2018.

2. Smith $M$, Saad $F$, Chowdhury $S$, et al. Apalutamide treatment and metastasis-free survival in prostate cancer. N Engl J Med 2018; 378:1408-1418. https://doi.org/10.1056/NEJMoal715546

3. Hussain M, Fizazi K, Saad F, et al. PROSPER: A phase 3, randomized, double-blind, placebo (PBO)controlled study of enzalutamide (ENZA) in men with non-metastatic castration-resistant prostate cancer (MO CRPC). J Clin Oncol 2018;36:3. https://doi.org/10.1200/JC0.2018.36.6_suppl.3

4. Boevé L, Hulshof M, Vis A, et al. PD10-10 A prospective, randomized controlled trial evaluating overall survival in patients with primary bone metastatic prostate cancer (MPCA) receiving either androgendeprivation therapy (ADT) or ADT combined with concurrent radiation therapy to the prostate, final data from the HORRAD trial. J Urol 2018;199:e231-2. https://doi.org/10.1016/i.juro.2018.02.620

Correspondence: Dr. Taehyoung Lee, McMaster University, Hamilton, 0N, Canada; Hee@qmed.ca 